\title{
Mycobacterium lepromatosis Lepromatous Leprosy in US Citizen Who Traveled to Disease-Endemic Areas
}

\author{
Abinash Virk, Bobbi Pritt, Robin Patel, \\ James R. Uhl, Spencer A. Bezalel, \\ Lawrence E. Gibson, Barbara M. Stryjewska, \\ Margot S. Peters
}

We report Mycobacterium lepromatosis infection in a US-born person with an extensive international travel history. Clinical symptoms, histopathology, and management are similar to those of infections caused by $M$. leprae. Clinicians should consider this pathogen in the diagnosis of patients with symptoms of leprosy who have traveled to endemic areas.

\begin{abstract}
A 59-year-old white man born in the United States came to the Travel and Tropical Medicine Clinic at Mayo Clinic (Rochester, Minnesota, USA) in March 2017 reporting 12 months of progressive skin lesions and prior onset of peripheral neuropathy and arthritis. The lesions started on his forearms, then progressed to nearly diffuse involvement of his face, neck, ears, and trunk. The lesions were not pruritic, painful, or ulcerative. Two years earlier, arthralgia of his hips, knees, and hands led to a diagnosis of rheumatoid arthritis on the basis of positive results for rheumatoid factor, negative cyclic citrullinated peptide antibody, and antinuclear antibodies. He received prednisone for several weeks, then methotrexate $(20 \mathrm{mg} / \mathrm{wk})$. He also had an electromyography-confirmed sensorimotor peripheral neuropathy that began 1 year before this presentation. After an unsuccessful empiric course of topical corticosteroid therapy, skin biopsies were collected from his neck and forearm; results showed sheets of histiocytes and scattered well-formed granulomas with perineural involvement. Fite stain was positive for numerous intracellular acid-fast bacilli, leading to a diagnosis of lepromatous leprosy; the patient was referred to the Division of Infectious Diseases at Mayo Clinic for further evaluation.

The patient was an administrator who worked indoors and did not have substantive outdoor exposure. He was born and raised in the United States but had an extensive travel history as an adult to many countries over several

Author affiliations: Mayo Clinic, Rochester, Minnesota, USA

(A. Virk, B. Pritt, R. Patel, J.R. Uhl, S.A. Bezalel, L.E. Gibson,

M.S. Peters); National Hansen's Disease Programs, Baton Rouge,

Louisiana, USA (B.M. Stryjewska)

DOI: https://doi.org/10.3201/eid2311.171104
\end{abstract}

decades, including 2 trips to the Pacific coast of Mexico (7 days each in Puerto Vallarta, Jalisco, in April 2005, and Acapulco in March 2007), a region to which Mycobacterium lepromatosis leprosy is endemic (1). He had no known exposure to a person with leprosy or to armadillos, which are known vectors for leprosy.

On physical examination, we found nearly diffuse erythema and induration of the patient's face, ears, neck (Figure 1), and chest, as well as his upper extremities, more focally involving the dorsal aspects of the forearms. He had partial loss of eyebrows bilaterally. The right auricle had a small, crusted ulcer. His nasal mucosa was thickened and nasal passages almost blocked. Ulnar and superficial cervical nerves were not easily palpated. He showed no clinical signs of active synovitis.

Initial laboratory test results were within reference ranges or negative, including complete blood count, erythrocyte sedimentation rate, C-reactive protein, creatinine, QuantiFERON-TB Gold In-Tube Test (Quest Diagnostics, Madison, NJ, USA), HIV serologies, rhumatoid factor (despite positive test 2 years prior), and cyclic citrullinated peptide antibodies. Chest radiograph results were unremarkable.

Skin biopsies of the neck, chin, and forearm showed patchy or diffuse granulomatous dermal inflammation, with foamy and epithelioid histiocytes. We found numerous acid-fast bacilli within histiocytes and invading nerves (Figure 2), highlighted by Fite, Gomori methenamine silver, and Gram stains; Ziehl-Neelsen stain highlighted only a few organisms. The combination of clinical, histopathologic, and histochemical staining features was diagnostic for multibacillary lepromatous leprosy.

We achieved a diagnosis by using the broad-range $16 \mathrm{~S}$ ribosomal RNA gene PCR assay on the formalinfixed, paraffin-embedded block of the chin biopsy, as follows: specimens were lysed with proteinase $K$, then incubated with $0.1-\mathrm{mm}$ silica beads in a thermomixer at $100^{\circ} \mathrm{C}$ with rapid mixing. DNA was extracted from the lysate with the Genomic DNA Clean \& Concentrator 10 kit (Zymo Research, Irvine, CA, USA). We used PCR with 5 $\mu \mathrm{L}$ of the DNA extract and previously described primers (2) on a Roche LightCycler 480 (Roche Molecular Systems Inc., Branchburg, NJ, USA) with SYBR Green stain. The PCR target is an $\approx 400$-base-pair portion, including the V3-V4 region, of the $16 \mathrm{~S}$ ribosomal RNA gene. The 

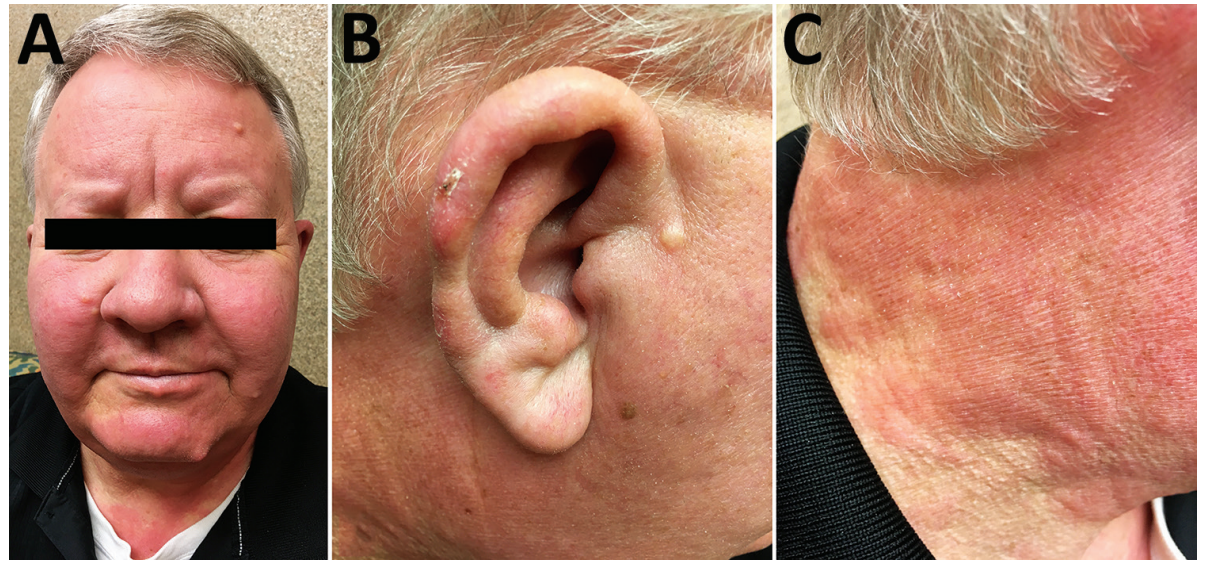

Figure 1. Signs of Mycobacterium lepromatosis infection in 59-year-old white male US citizen, 2017. A) Leonine facies with partial loss of eyebrows and nodular lesion of chin. B) Right ear nodularity with focal crusted ulceration. C) Confluent erythema from face to neck. amplified product, which we sequenced by using Sanger sequencing, showed identical nucleotides to $M$. lepromatosis strain FJ924 (positions 368-765, GenBank accession no. EU203590) and a 3-nt difference from Mycobacterium leprae Br4923 (GenBank accession no. FM211192).

The presence of $M$. lepromatosis was also confirmed by using M. lepromatosis-specific PCR at the National Hansen's Disease Program (Baton Rouge, LA, USA). In consultation with this program, we prescribed clarithromycin, rifampin, and dapsone in April 2017. Within 3 months of treatment, the patient had decreased skin induration, nasal obstruction, and pinna thickening but minimal improvement in arthralgias or peripheral neuropathy symptoms. No immune reactions occurred during treatment.

Until the advent of molecular methods, all leprosy worldwide was assumed to have been caused by M. leprae. In 2008, a novel Mycobacterium species, M. lepromatosis, was identified by multigene analysis on tissue obtained from 2 immigrants to the United States from Mexico who died from diffuse lepromatous leprosy (3), which is endemic to Mexico and Costa Rica and is rarely reported from other geographic locations (4).

Comparative genomics show that M. leprae and M. lepromatosis are closely related and derived from a common ancestor (4) with $\approx 9.1 \%$ genetic difference between them, suggesting species-level divergence of $\approx 10$ million (5) to 13.9 million (4) years ago. A phylogeographic survey suggests that M. lepromatosis is not widespread (4). Since 2008, PCR testing of patients identified M. lepromatosis from Mexico $(1,6)$, Singapore (7), Canada (8), and Myanmar and Brazil (9).

Unlike M. leprae (10), M. lepromatosis has not been found in armadillos. Red squirrels are infected with M. lepromatosis in the United Kingdom (11), but the geographic distribution, zoonotic transmission risks, and other animal reservoirs are unknown.

Excluding the patient we report, $M$. lepromatosis lepromatous leprosy has been diagnosed in 10 persons (including the 2 index case-patients) in the United States: all were immigrants (8 from Mexico, 2 from Costa Rica) $(3,6,12)$. Our report emphasizes that US citizens can acquire M. lepromatosis when traveling to Mexico or other locations as tourists. Our patient's earliest symptoms of arthritis and neuropathy were in December 2014, suggesting acquisition in Puerto Vallarta (2005) or Acapulco (2007), consistent with the leprosy incubation period of 7-8 years (6). His signs and symptoms were similar to those of patients from Mexico $(1,6,12,13)$ and a patient from Canada in whom peripheral neuropathy developed 1-2 years before onset of skin lesions. Nasal mucosal

Figure 2. Skin biopsies of 59-year-old white male US citizen showing Mycobacterium lepromatosis infection, 2017. A) Hematoxylin and eosin-stained section of a specimen from the chin showing granulomatous dermal inflammation (original magnification $\times 100$ ); inset shows nerve involvement (arrows) that is diagnostic for leprosy (original magnification $\times 400$ ). B) Fitestained section of a specimen from the chin highlights

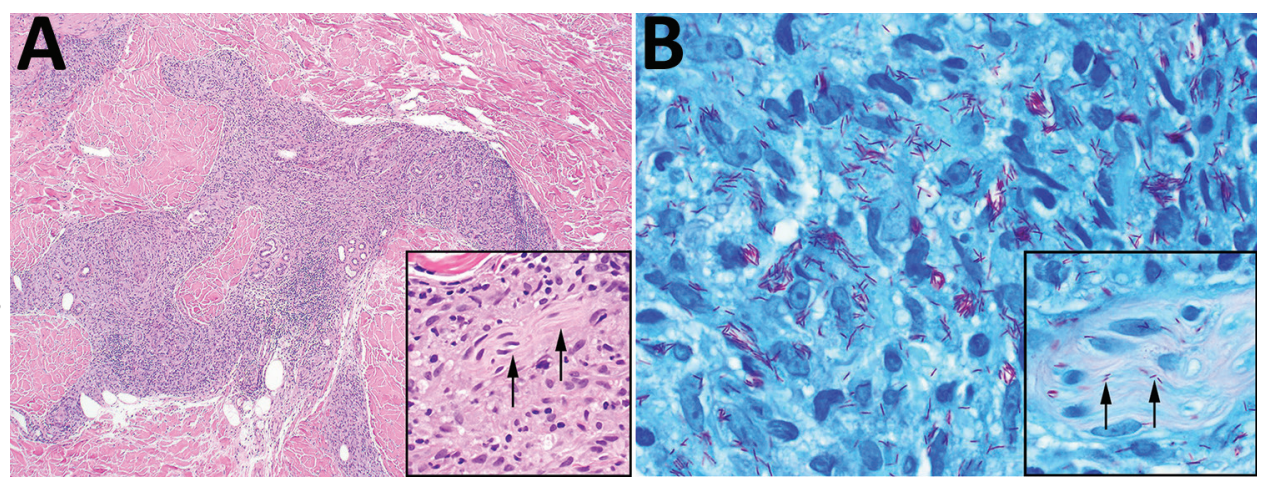
numerous acid-fast bacilli within histiocytes (original magnification $\times 1,000$ ); inset shows peripheral nerve involvement (arrows) that is diagnostic for leprosy (original magnification $\times 1,000$ ). 
involvement was prominent in both. Loss of eyebrows, eyelashes, or both is common in $M$. lepromatosis infection $(6,8,13)$ and might be an early sign of infection. Future reports may help determine if this feature is specific for $M$. lepromatosis. In the case reported by Han et al. (13), cure was associated with nearly complete regrowth of eyebrows and eyelashes.

The full spectrum of manifestations, outcomes, and global burden of $M$. lepromatosis infection remains unknown. In a study of persons who had leprosy, specifically diffuse lepromatous leprosy in Mexico, M. lepromatosis was identified more often $(63.2 \%)$ than $M$. leprae and caused dual infections in $16.1 \%(1)$.

Leprosy can resemble autoimmune disorders, including systemic lupus erythematosus or rheumatoid arthritis. Chronic polyarthritis is described in up to $75 \%$ of leprosy patients, possibly secondary to an immune response to mycobacterial heat-shock proteins $(14,15)$. Overlapping occurrences of rheumatoid and leprous arthritis make it difficult to differentiate these disorders. The arthritis in the patient we report could be leprous.

Reported patients who have M. lepromatosis and $M$. leprae lepromatous leprosy have been treated similarly (12). Because of the multibacillary load and higher risk for immune reactions and pigmentation, especially with clofazimine or minocycline, we prescribed clarithromycin, rifampin, and dapsone for this patient. He was maintained, at our advice, on methotrexate to modulate these reactions.

In summary, $M$. lepromatosis lepromatous leprosy is a travel-related hazard for travelers to Mexico or other disease-endemic areas. Specific exposure risks for acquisition of $M$. lepromatosis are unknown. The presence of leprosylike skin lesions should prompt detailed evaluation, including skin biopsy for histopathology, histochemical stains for mycobacterial organisms, and $16 \mathrm{~S}$ ribosomal RNA gene PCR to identify the causative agent.

A.V. is an inventor for Travel Health and Wellness, LLC; R.P. reports grants from CD Diagnostics, BioFire, Curetis, Merck, Hutchison Biofilm Medical Solutions, Accelerate Diagnostics, Allergan, and The Medicines Company. R.P. is a consultant to Curetis; monies are paid to Mayo Clinic. In addition, R.P. has a patent on Bordetella pertussis/parapertussis PCR issued; a patent on a device/method for sonication with royalties paid by Samsung to Mayo Clinic; and a patent on an anti-biofilm substance issued. R.P. serves on an Actelion data monitoring board; receives travel reimbursement from Roche, ASM, and IDSA; an editor's stipend from ASM and IDSA; and honoraria from the NBME, Up-to-Date, and the Infectious Diseases Board Review Course.

Dr. Virk has been a consultant in the Division of Infectious Diseases at the Mayo Clinic in Rochester, Minnesota, since 1997. She is the chair of the Enterprise Antimicrobial Stewardship Program at the Mayo Clinic and previously was the director of the Travel and Tropical Medicine Clinic.

\section{References}

1. Han XY, Sizer KC, Velarde-Félix JS, Frias-Castro LO, Vargas-Ocampo F. The leprosy agents Mycobacterium lepromatosis and Mycobacterium leprae in Mexico. Int J Dermatol. 2012;51:952-9. http://dx.doi.org/10.1111/ j.1365-4632.2011.05414.x

2. Gomez E, Cazanave C, Cunningham SA, Greenwood-Quaintance KE, Steckelberg JM, Uhl JR, et al. Prosthetic joint infection diagnosis using broad-range PCR of biofilms dislodged from knee and hip arthroplasty surfaces using sonication. J Clin Microbiol. 2012;50:3501-8. http://dx.doi.org/10.1128/JCM.00834-12

3. Han XY, Seo YH, Sizer KC, Schoberle T, May GS, Spencer JS, et al. A new Mycobacterium species causing diffuse lepromatous leprosy. Am J Clin Pathol. 2008;130:856-64. http://dx.doi.org/10.1309/AJCPP72FJZZRRVMM

4. Singh P, Benjak A, Schuenemann VJ, Herbig A, Avanzi C, Busso P, et al. Insight into the evolution and origin of leprosy bacilli from the genome sequence of Mycobacterium lepromatosis. Proc Natl Acad Sci U S A. 2015;112:4459-64. http://dx.doi.org/10.1073/pnas.1421504112

5. Han XY, Sizer KC, Thompson EJ, Kabanja J, Li J, Hu P, et al. Comparative sequence analysis of Mycobacterium leprae and the new leprosy-causing Mycobacterium lepromatosis. J Bacteriol. 2009;191:6067-74. http://dx.doi.org/10.1128/JB.00762-09

6. Sotiriou MC, Stryjewska BM, Hill C. Two cases of leprosy in siblings caused by Mycobacterium lepromatosis and review of the literature. Am J Trop Med Hyg. 2016;95:522-7. http://dx.doi.org/10.4269/ajtmh.16-0076

7. Han XY, Sizer KC, Tan HH. Identification of the leprosy agent Mycobacterium lepromatosis in Singapore. J Drugs Dermatol. 2012;11:168-72. PubMed

8. Jessamine PG, Desjardins M, Gillis T, Scollard D, Jamieson F, Broukhanski G, et al. Leprosy-like illness in a patient with Mycobacterium lepromatosis from Ontario, Canada. J Drugs Dermatol. 2012;11:229-33. PubMed

9. Han XY, Aung FM, Choon SE, Werner B. Analysis of the leprosy agents Mycobacterium leprae and Mycobacterium lepromatosis in four countries. Am J Clin Pathol. 2014;142:524-32. http://dx.doi.org/10.1309/AJCP1GLCBE5CDZRM

10. Truman RW, Singh P, Sharma R, Busso P, Rougemont J, Paniz-Mondolfi A, et al. Probable zoonotic leprosy in the southern United States. N Engl J Med. 2011;364:1626-33. http://dx.doi.org/10.1056/NEJMoa1010536

11. Avanzi C, Del-Pozo J, Benjak A, Stevenson K, Simpson VR, Busso P, et al. Red squirrels in the British Isles are infected with leprosy bacilli. Science. 2016;354:744-7. http://dx.doi.org/10.1126/ science.aah3783

12. Han XY, Jessurun J. Severe leprosy reactions due to Mycobacterium lepromatosis. Am J Med Sci. 2013;345:65-9. http://dx.doi.org/10.1097/MAJ.0b013e31826af5fb

13. Han XY, Quintanilla M. Diffuse lepromatous leprosy due to Mycobacterium lepromatosis in Quintana Roo, Mexico. J Clin Microbiol. 2015;53:3695-8. http://dx.doi.org/10.1128/ JCM.01951-15

14. Henriques CC, Lopez B, Mestre T, Grima B, Panarra A, Riso N. Leprosy and rheumatoid arthritis: consequence or association? BMJ Case Rep. 2012;2012. http://dx.doi.org/10.1136/ bcr.12.2011.5346

15. Atkin SL, el-Ghobarey A, Kamel M, Owen JP, Dick WC. Clinical and laboratory studies of arthritis in leprosy. BMJ. 1989;298:1423-5. http://dx.doi.org/10.1136/bmj.298.6685.1423

Address for correspondence: Abinash Virk, Division of Infectious Diseases, Mayo Clinic, 200 First St SW, Rochester, MN 55905, USA; email: virka@mayo.edu 